Ophthalmologe 2022 $\cdot 119: 491-496$ https://doi.org/10.1007/s00347-021-01527-w Eingegangen: 26. Juli 2021 Überarbeitet: 12. Oktober 2021 Angenommen: 15. Oktober 2021 Online publiziert: 4. November 2021 (c) Der/die Autor(en) 2021

\section{iOCT in der klinischen Anwendung}

\section{Korrelation von intraoperativer Morphologie und postoperativem Ergebnis bei Patienten mit durchgreifendem Makulaforamen}

Hintergrund: Die Beurteilung der intraoperativen Veränderung der Netzhautmorphologie, insbesondere des vitreoretinalen Überganges, ist mithilfe der intraoperativen optischen Kohärenztomografie (iOCT) möglich geworden.

Ziel der Arbeit: Um die Bedeutung der intraoperativen Morphologie beim durchgreifenden Makulaforamen (MF) für das postoperative funktionelle Ergebnis zu evaluieren, wurde eine retrospektive, klinische Beobachtungsstudie durchgeführt. Material und Methoden: Die Netzhautmorphologie wurde in 32 Augen von 32 konsekutiven Patienten mit durchgreifendem Makulaforamen mittels iOCT zu verschiedenen Zeitpunkten während der Operation beobachtet. Die Veränderungen wurden anschließend mit dem postoperativen funktionellen Ergebnis korreliert. Ergebnisse: Nach Induktion der hinteren Glaskörperabhebung (HGA) reduzierte sich der Makulaforamen-Index (MHI) um $-0,05(p=0,01)$, die basale Foramenbreite (FB) stieg um $+99,4 \mu \mathrm{m}(\mathrm{SD}=197,8 \mu \mathrm{m} ; p=0,04)$. Die Verschlussrate betrug $100 \%$ zum Zeitpunkt der ersten postoperativen Vorstellung nach im Mittel 73 Tagen, der postoperative Visus verbesserte sich signifikant $(p<0,05)$.

Es zeigte sich eine signifikant positive Korrelation von intraoperativer Morphologie und postoperativem Ergebnis zwischen einem niedrigen $\mathrm{MHI}$ und einem besseren postoperativen Visus ( $S K K=0,50 ; p=0,02)$, zwischen einer großen FB und einem besseren postoperativen Visus (SKK $=0,43 ; p=0,05$ ) sowie zwischen einer breiten Apertur nach HGA und einem größeren Visusanstieg postoperativ ( $\mathrm{SKK}=0,44 ; p=0,03$ ). Diskussion: Wir konnten eine Abflachung sowie eine Verbreiterung des MF durch Lösen der vitreoretinalen Zugkräfte beobachten. Aufgrund des Zusammenhangs zwischen einer großen intraoperativen FB mit einem besseren postoperativen Visus scheint die intraoperative Relaxierung der Netzhaut bedeutsam.

\section{Schlüsselwörter}

IOCT · Operative Bildgebung · Vitreoretinalchirurgie · Makulaforamen · SD-OCT

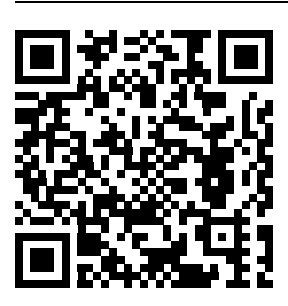

QR-Code scannen \&Beitrag online lesen

\section{Hintergrund und Fragestellung}

Durch die Integration des OCT-Gerätes in das Operationsmikroskop konnte die intraoperative Beobachtung mikrostruktureller Veränderungen der Netzhaut realisiert werden $[3,6,9,10,12]$.
Neben Alter, Visus und MF-Größe als präoperative prognostische Faktoren [11, $19,21]$ scheint der präoperative Makulaforamen-Index (MHI) relevant für das postoperative funktionelle Ergebnis zu sein [14]. 


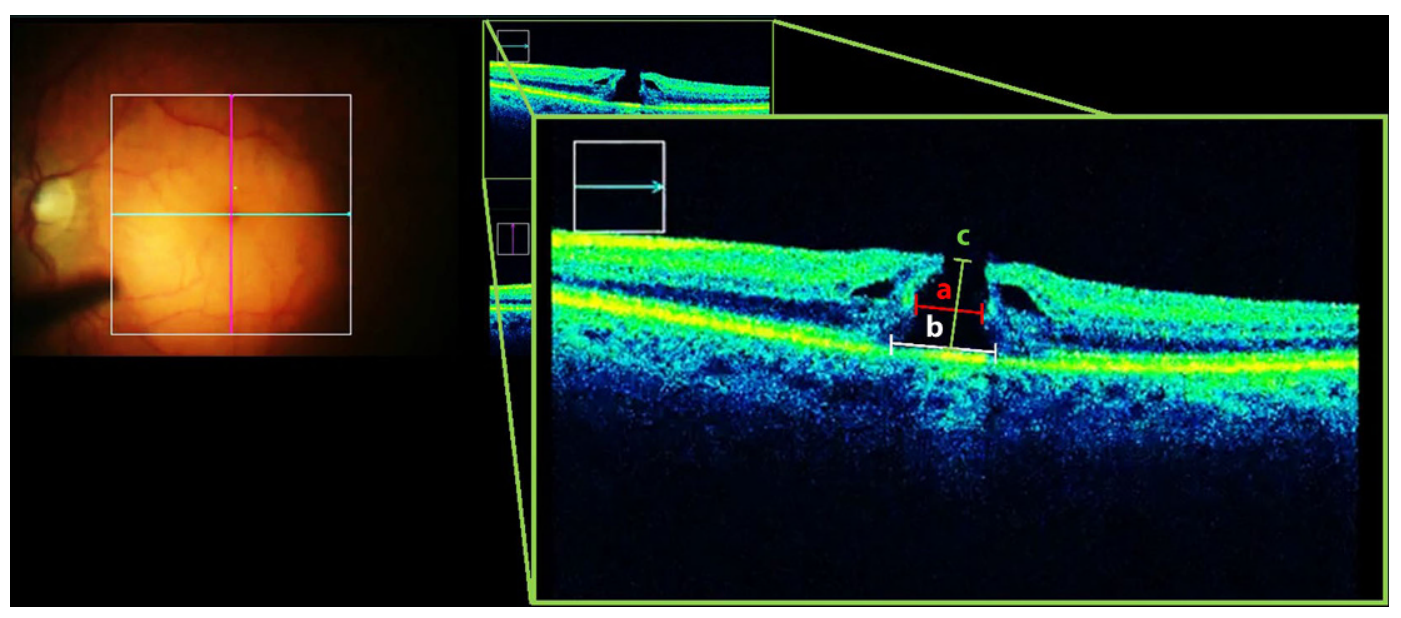

Abb. $1<$ Parameter zur Beschreibung der Makulaforamengeometrie mittels iOCT $(a=$ Apertur, $b=$ basale Foramenbreite $c=$ Netzhautdicke im Bereich der Foramenränder, $\mathrm{MHI}=\mathrm{c} / \mathrm{b}$ )
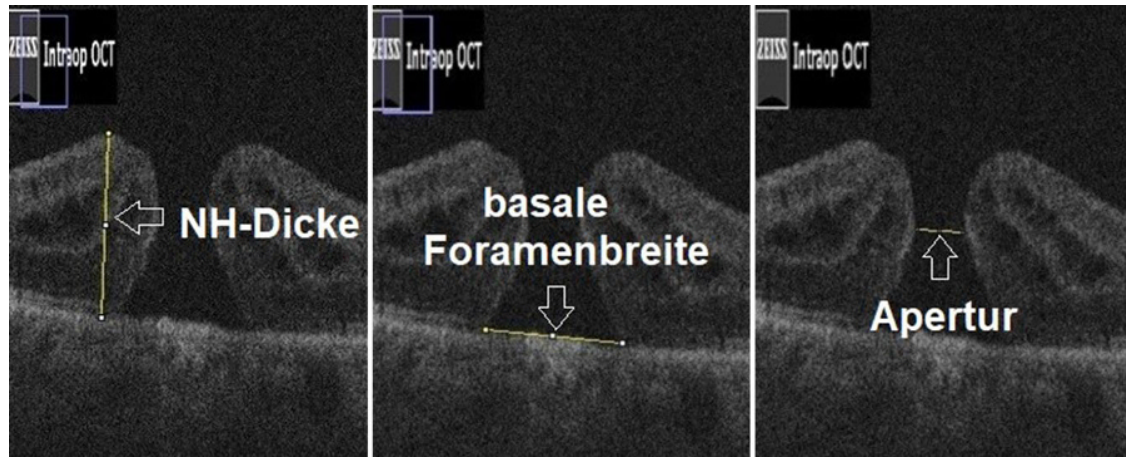

Abb. 2 ム Retrospektive Vermessung der intraoperativ gewonnenen iOCT-Volumenscans mithilfe der Software ImageJ

Das Ziel dieser Studie war die Evaluation der Netzhautmorphologie während chirurgischer Manipulation bei Patienten mit MF mittels intraoperativer OCT (iOCT) und der Korrelation dieser Veränderungen mit dem postoperativen Visusergebnis.

\section{Studiendesign und Unter- suchungsmethoden}

Zur Evaluation des IOCT in der klinischen Routine wurde die intraoperative Netzhautmorphologie von 32 Augen von 32 konsekutiven Patienten analysiert. Aufgrund unvollständiger Daten waren 14 Patienten ausgeschlossen worden. Eingeschlossen wurden alle Patienten, die aufgrund eines idiopathischen, durchgreifenden Makulaforamens im Zeitraum von Mai 2015 bis Dezember 2016 iOCT-assistiert operiert wurden. Komorbiditäten wie Netzhautablösung, Glaskörperblutung, vaskuläre Erkrankungen, altersabhängige Makuladegeneration, Glaukom und jegliche zuvor durchgeführte Netzhautoperation inklusive der medikamentenin- duzierten Vitreolyse mittels Ocriplasmin

Bestkorrigierter Visus, Linsenstatus und MF-Größe, gemessen an der Apertur, wurden präoperativ, im Zeitraum 3 Wochen bis 3 Monate postoperativ und nach mindestens 6 Monaten postoperativ evaluiert. Der Visus wurde standardmäßig in Dezimal erhoben, die Darstellung in dieser Arbeit erfolgt zur besseren Vergleichbarkeit umgerechnet in logMAR. Die MF-Apertur wurde präoperativ mithilfe des Heidelberg Eye Explorer (Heyex, Software Serial Number: H2E-18404-028-011, Heidelberg Engeneering, Heidelberg, Germany) vermessen.

Alle Patienten wurden mittels standardisierter transkonjunktivaler, nahtloser 23-Gauge-Pars-plana-Vitrektomie (ppV), iOCT- sowie färbungsgestütztem Membranpeeling der internen limitierenden Membran (ILM) (G-81005 Brilliant Peel @); Fluoron, Geuder AG, Heidelberg, Germany) sowie Tamponade eines Luft-GasGemisches (C3F8, 12\%) operiert. Die Analyse erfolgte anhand von intraoperativ gewonnenen iOCT-Volumenscans, aufstellten Ausschlusskriterien dar. genommen mit dem OPMI Lumera 700 Mikroskop (Carl Zeiss Meditec AG, Jena, Germany) mit integriertem RESCAN 700 IOCT (SDOCT, 27000 A-Scans pro Sekunde, Wellenlänge $840 \mathrm{~nm}$, A-Scan Tiefe 2,0 mm, axiale Auflösung 5,5 $\mu \mathrm{m}$ [2]).

Die Operation wurde jeweils vom gleichen Ophthalmochirurgen (M. M.) durchgeführt, und die Auswertung der intraoperativen Morphologie bezog sich auf IOCTDaten zu folgenden Zeitpunkten: zu Beginn der Operation, nach Induktion der hinteren Glaskörperabhebung (HGA) und nach ILM-Membranpeeling. Die Daten dieser iOCT-Scans bildeten die Grundlage für die retrospektive Auswertung.

Die retrospektive Vermessung der MFMorphologie im intraoperativ gewonnenen iOCT-Volumenscan erfolgte mithilfe der Software ImageJ (freeware, National Institutes of Health, Bethesda, MD, USA).

Die MF-Geometrie wurde dann anhand der Apertur, der basalen Foramenbreite (FB), der Netzhautdicke im Bereich der MF-Ränder sowie des MHI bestimmt. Die Netzhautmorphologie aus Sicht des Operateurs im iOCT ist in $\mathbf{A b b} \mathbf{1}$ dargestellt. Die retrospektive Vermessung der Parameter mithilfe der Software ImageJ ist in

- Abb. 2 veranschaulicht.

Die Apertur ist definiert als geringster Durchmesser des MF, gemessen auf Höhe der mittleren Netzhautschichten parallel zum retinalen Pigmentepithel (RPE) [4]. Die FB ist definiert als breitester Durchmesser des MF direkt über dem RPE gemessen, die Netzhautdicke im Bereich der MF-Ränder ist definiert als die größte Höhe des MF, gemessen von der ILM bis zum RPE, und der MHI errechnet sich aus dem Ver- 


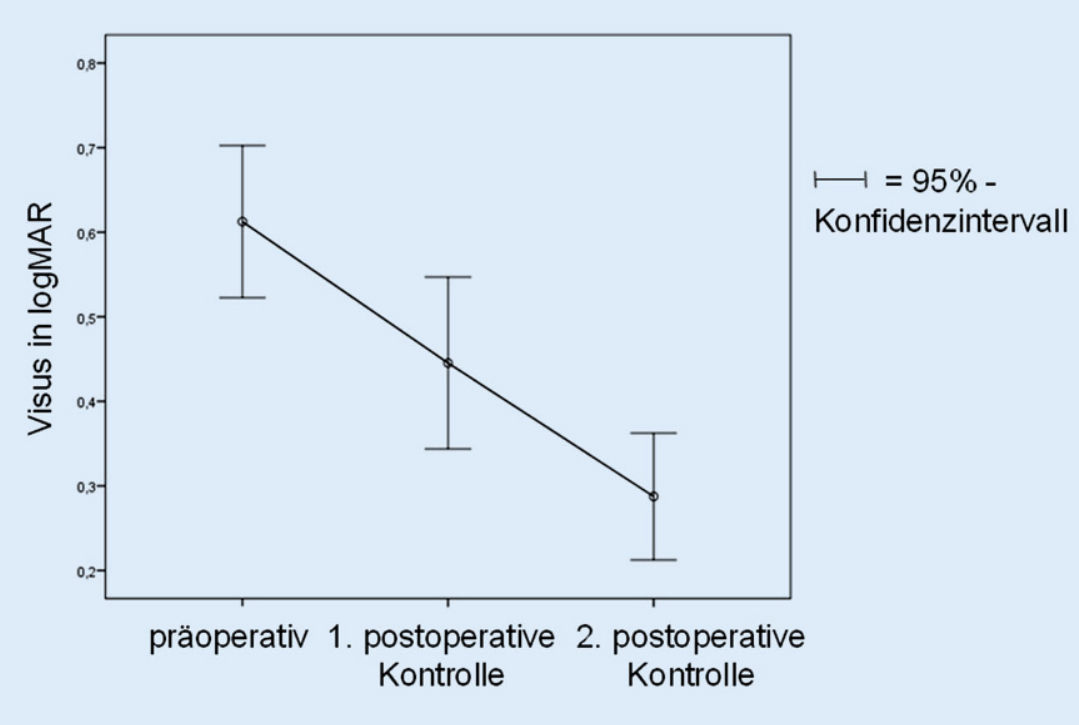

Abb. 3 A Visusverlauf. „1. postoperative Kontrolle“ = mittlerer Visus 3 Wochen bis 3 Monate postoperativ; , 2. postoperative Kontrolle" $=$ mittlerer Visus mindestens 6 Monate postoperativ

hältnis der Netzhautdicke und der basalen Foramenbreite [14].

Die statistische Auswertung wurde mit der Software IBM SPSS Statistics Version 22 (IBM, New York, USA) auf einem Signifikanzniveau von $p=0,05$ durchgeführt.

Es besteht ein Zulassungsvotum der Ethikkommission der TU München.

\section{Ergebnisse}

Die Geschlechterverteilung innerhalb der 32 Patienten zeigte ein Verhältnis von annähernd 3,5:1 mit 25 (78\%) weiblichen und 7 (22\%) männlichen Patienten. Das durchschnittliche Alter betrug 69 Jahre $(S D=8,4$ Jahre).

Der mittlere Visus betrug präoperativ 0,61 logMAR ( $S D=0,25 \log M A R)$ mit einer Pseudophakierate von 11 (34\%) Patienten. Die mittlere MF-Größe, gemessen an der Apertur, betrug präoperativ 332,6 $\mu \mathrm{m}$ $(S D=152,1 \mu \mathrm{m})$ mit einer Größenverteilung in kleines MF $(<250 \mu \mathrm{m}-34,5 \%$, $n=11)$, mittelgroßes MF $(250-400 \mu \mathrm{m}-$ $31 \%, n=10)$ und großes MF $(>400 \mu \mathrm{m}-$ $34,5 \%, n=11$ ) von jeweils annähernd einem Drittel [4].

Bei 8 (25\%) Patienten konnte im präoperativen SD-OCT eine vitreomakuläre Adhäsion, bei 12 (38\%) Patienten eine vitreopapilläre Adhäsion und bei 11 (34\%) Patienten eine komplette hintere Glaskörperabhebung nachgewiesen werden. Bei einem (3\%) Patienten konnte der vitreoretinale Übergang im präoperativen SD-OCT aufgrund mangelnder Bildqualität nicht beurteilt werden.

Nach HGA zeigten sowohl die Apertur (MW: $-24,1 \mu \mathrm{m} ; \mathrm{SD}=97,8 \mu \mathrm{m} ; p=0,27$ ) als auch die Netzhautdicke im Bereich der MF-Ränder (MW: $-19,6 \mu \mathrm{m}$; SD = 72,2 $\mu \mathrm{m}$; $p=0,24$ ) eine Abnahmetendenz. Die FB nahm nach $H G A$ signifikant um $+99,4 \mu \mathrm{m}$ zu (SD = 197,8 $\mu \mathrm{m} ; p=0,04)$, und der MHI reduzierte sich signifikant um $-0,45$ (Minimum $=-1,7$; Maximum $=+0,1 ; p=0,01$ ). Die Reduktion des MHI stellt eine Abnahme der Foramenhöhe in Relation zur basalen Foramenbreite dar.

Nach ILM-Membranpeeling zeigten sowohl die Apertur (MW: $-40,5 \mu \mathrm{m}$; $\mathrm{SD}=117,8 ; p=0,14)$ als auch die FB (MW: $-56,8 \mu \mathrm{m} ; \mathrm{SD}=369,6 \mu \mathrm{m} ; p=0,52$ ) eine Reduktionstendenz. Die Netzhautdicke im Bereich der MF-Ränder (MW: $+24,7 \mu \mathrm{m}$; $\mathrm{SD}=62,5 \mu \mathrm{m} ; p=0,11$ ) sowie der $\mathrm{MHI}$ (MW: $+0,02 ; \mathrm{SD}=0,13 ; p=0,53$; t-Test) zeigten eine Zunahmetendenz, was eine Zunahme der Foramenhöhe im Vergleich zur basalen Foramenbreite widerspiegelt.

Die erste postoperative Datenerhebung erfolgte nach einer mittleren Zeit von 73 Tagen (SD=63 Tage), und zu diesem Zeitpunkt zeigte sich eine MFVerschlussrate von $100 \%$. Die Zeitspanne zwischen Operation und zweiter postoperativer Datenerhebung betrug im
Mittel 297 Tage (SD = 118 Tage). Der Visus zum Zeitpunkt der ersten postoperativen Datenauswertung betrug 0,45 logMAR ( $S D=0,28$ logMAR) und zeigte sich damit im Vergleich zum präoperativen Visus $(0,61 \log M A R ; S D=0,25 \log M A R)$ signifikant besser $(-0,16 \log M A R ; S D=0,28$; $p<0,01)$. Der Visus zum Zeitpunkt der zweiten postoperativen Auswertung betrug 0,29 $\log M A R(S D=0,21 \log M A R)$ und war damit ebenfalls signifikant besser als der präoperative Visus $(-0,32$ logMAR; $\mathrm{SD}=0,27 ; p<0,01)$. Der Visusverlauf ist in - Abb. 3 dargestellt.

Die Korrelation von intraoperativen Morphologieparametern (Apertur, Netzhautdicke im Bereich der MF-Ränder, FB, MHI) und Visus zum Zeitpunkt der ersten postoperativen Datenauswertung (73 Tage) ergab keine signifikanten $\mathrm{Zu}$ sammenhänge.

Die Zusammenhänge von intraoperativer Morphologie und Visus zum Zeitpunkt der zweiten postoperativen Datenauswertung sind in • Tab. 1 dargestellt.

Die Analyse in Bezug auf die relative postoperative Visusveränderung im Vergleich zum präoperativen Visus zeigte eine signifikante Korrelation zwischen einer breiten Apertur nach HGA und einer größeren Visusverbesserung postoperativ in unserer Kohorte von 32 Augen (SKK= 0,44; $p=0,03)$ (• Tab. 2).

Des Weiteren zeigten sich eine signifikante Korrelation zwischen einer großen basalen Foramenbreite nach ILM-Membranpeeling und einem besseren postoperativen Visus (SKK $=-0,43 ; p=0,05$ ) (- Abb.4) sowie eine signifikante Korrelation zwischen einem niedrigen $\mathrm{MHI}$ nach ILM-Membranpeeling und einem besseren postoperativen Visus $(\mathrm{SKK}=0,50$; $p=0,02$ ) (• Abb.5). Dies weist darauf hin, dass ein niedriger intraoperativer $\mathrm{MHI}$, welcher eine große FB im Verhältnis zur Netzhautdicke im Bereich der MF-Ränder widerspiegelt, ein positiver prognostischer Marker für den postoperativen Visus ist (- Tab. 3).

\section{Diskussion}

Unsere Beobachtungen zeigen eine signifikante Zunahme der FB und eine signifikante Abnahme des MHI nach HGA. Die Breitenzunahme und Abflachung des 
Tab. 1 Korrelation von intraoperativer MF-Morphologie und Visusergebnis (mindestens 6 Monate postoperativ)

\begin{tabular}{|l|l|l|l|l|l|l|}
\hline & \multicolumn{3}{|l|}{$\begin{array}{l}\text { Zu Beginn der Opera- } \\
\text { tion }\end{array}$} & \multicolumn{2}{l|}{$\begin{array}{l}\text { Nach Induktion der } \\
\text { HGA }\end{array}$} & \multicolumn{2}{l|}{$\begin{array}{l}\text { Nach ILM-Membran- } \\
\text { peeling }\end{array}$} \\
\hline & SKK & Signifikanz & SKK & Signifikanz & SKK & Signifikanz \\
\hline Visus - Apertur & $-0,34$ & $p=0,11$ & $-0,15$ & $p=0,49$ & $-0,25$ & $p=0,26$ \\
\hline $\begin{array}{l}\text { Visus - basale } \\
\text { Foramenbreite }\end{array}$ & $-0,11$ & $p=0,61$ & $-0,16$ & $p=0,48$ & $-0,43$ & $p=0,05$ \\
\hline $\begin{array}{l}\text { Visus - Netzhaut- } \\
\text { dicke }\end{array}$ & 0,26 & $p=0,24$ & 0,28 & $p=0,20$ & 0,14 & $p=0,53$ \\
\hline Visus - MHI & 0,20 & $p=0,35$ & 0,20 & $p=0,35$ & 0,50 & $p=0,02$ \\
\hline SKK Spearman-Korrelationskoeffizient & \multicolumn{7}{ll}{} \\
\hline
\end{tabular}

Tab. 2 Korrelation von intraoperativer MF-Morphologie und relativer postoperativer Visusverbesserung im Vergleich zum präoperativen Visus

\begin{tabular}{|l|l|l|}
\hline & \multicolumn{2}{l|}{ Nach Induktion der HGA } \\
\hline & SKK & Signifikanz \\
\hline Visusverbesserung - Apertur & 0,44 & $p=0,03$ \\
\hline Visusverbesserung - basale Foramenbreite & 0,20 & $p=0,37$ \\
\hline Visusverbesserung - Netzhautdicke & 0,23 & $p=0,30$ \\
\hline Visusverbesserung - MHI & $-0,19$ & $p=0,38$ \\
\hline SKK Spearman-Korrelationskoeffizient & \multicolumn{2}{l}{} \\
\hline
\end{tabular}

Tab. 3 Vergleich der beiden Gruppen (intraoperativer $\mathrm{MHI}<0,5 / \geq 0,5$ ) bezogen auf das postoperative Visusergebnis (mindestens 6 Monate postoperativ)

\begin{tabular}{|l|l|l|l|}
\hline & Signifikanz & MHI $<0,5$ & MHI $\geq 0,5$ \\
\hline MHI zu Beginn der Operation & $p=0,90$ & $n=12$ & $n=11$ \\
\hline MHI nach Induktion der HGA & $p=0,93$ & $n=16$ & $n=8$ \\
\hline MHI nach ILM-Membranpeeling & $p=0,06$ & $n=14$ & $n=9$ \\
\hline
\end{tabular}

MF interpretieren wir als Ausdruck einer Entspannung der Netzhaut aufgrund der Reduktion vitreoretinaler Zugkräfte. Bezogen auf die Apertur und die FB verkleinert sich das MF nach ILM-Membranpeeling am ehesten durch das Nachlassen tangentialer Zugkräfte der ILM auf die Netzhaut. In unserer Kohorte von 32 Augen zeigte sich ein signifikanter Zusammenhang zwischen einer intraoperativ großen FB und einem besseren Visusergebnis sowie zwischen einem intraoperativ niedrigen $\mathrm{MHI}$ und einem besseren Visusergebnis. Wir interpretieren ein intraoperativ verbreitetes und abgeflachtes MF als einen guten prognostischen Faktor.

Sowohl der Zusammenhang einer großen FB nach ILM-Membranpeeling als auch einer breiteren Apertur nach HGA mit einem besseren postoperativen Visus zeigt, dass die intraoperative Reduktion von zuvor bestehenden Zugkräften auf den vitreoretinalen Übergang und die damit verbundene Entspannung der Netzhaut sehr bedeutsam für das postoperative funktionale Ergebnis ist. Bereits 2014 beobachteten Ehlers et al. einen signifikanten Zusammenhang zwischen der intraoperativen Veränderung der basalen Foramenbreite nach ILM-Membranpeeling und der MF-Verschlussrate $(p=0,01)$ [8]. Meyer et al. beschrieben 2017 die Lösung der Verzahnung von Photorezeptoren und RPE durch subretinale Applikation von Flüssigkeit. Die so induzierte Mobilität der Netzhaut fördert den MF-Verschluss [18]. Ehlers et al. beschrieben eine intraoperative Expansion zwischen EZ und RPE als zunehmende Netzhautmobilität [7]. In Analogie hierzu könnten eine intraoperative Zunahme der Apertur und der FB ebenfalls Ausdruck der Netzhautmobilität sein. Durch reduzierte retinale Adhärenz werden die Readaptation der Foramenränder und ein zügiger Heilungsverlauf gefördert [16].

Kusuhara et al. wiesen 2004 bei Patienten mit einem präoperativen $\mathrm{MHI} \geq 0,5$ einen besseren postoperativen Visus nach als bei Patienten mit einem präoperativen $\mathrm{MHI}<0,5(p=0,03)$ [14]. Zu einem ähnlichen Ergebnis kamen wir in einer Langzeitnachverfolgung von Patienten mit MF, welche mithilfe der Inverted-Flap-Membranpeeling-Technik operiert wurden [1]. Beide Arbeiten verwendeten zur statistischen Auswertung die präoperativen $\mathrm{MHI}$ Daten. Im Gegensatz dazu zeigten die Ergebnisse der vorliegenden Arbeit eine signifikante Korrelation zwischen einem niedrigen intraoperativen $\mathrm{MHI}$ und einem besseren postoperativen Visus $(p=0,02)$. Der präoperative $\mathrm{MHI}$ scheint eine andere Aussagekraft zu haben als der intraoperativ erhobene $\mathrm{MHI}$. Die intraoperative Veränderung des MHI könnte ein weiteres Maß für die durch Meyer et al. beschriebene Mobilität der Netzhaut sein [18].

Eine Limitierung der vorliegenden Arbeit ist das retrospektive Studiendesign. Des Weiteren könnte das postoperative Visusergebnis von der Dauer der Erkrankung beeinflusst sein [13]. Da die Zeitspanne zwischen Erstauftreten von Symptomen und dem Operationszeitpunkt nicht erfasst wurde, war eine Analyse diesbezüglich retrospektiv nicht möglich.

Bei 6 (19\%) Patienten erfolgte intraoperativ ein ILM-Membranpeeling mit sog. Inverted-Flap-Technik [15], da es sich um Patienten mit sehr großem MF handelte. Das Ziel unserer Arbeit bestand in der Auswertung der klinischen Anwendung des iOCT anhand der Beobachtung der intraoperativen Morphologie. Unserer Ansicht nach wird die Analyse des Stellenwertes des IOCT nicht maßgeblich durch diese Operationstechnik beeinflusst, da der ILMFlap bei der sog. Cover-Technik [19] auf der Netzhaut positioniert, jedoch nicht in das MF hineingedrückt wird.

Präoperativ waren 11 (34\%) Patienten pseudophak. Dieser Anteil stieg zum Zeitpunkt der ersten postoperativen Analyse auf 17 (53\%) Patienten und zum Zeitpunkt der zweiten postoperativen Analyse auf 24 (75\%) Patienten an. Eine negative Beeinflussung des postoperativen Visus durch die mögliche Kataraktentwicklung bei den 8 ( $25 \%$ ) phaken Patienten mit einem mittleren Alter von 69 Jahren kann nicht ausgeschlossen werden. Die retrospektive Auswertung der Daten ließ keine Schweregradeinstufung der Katarakt zu. 


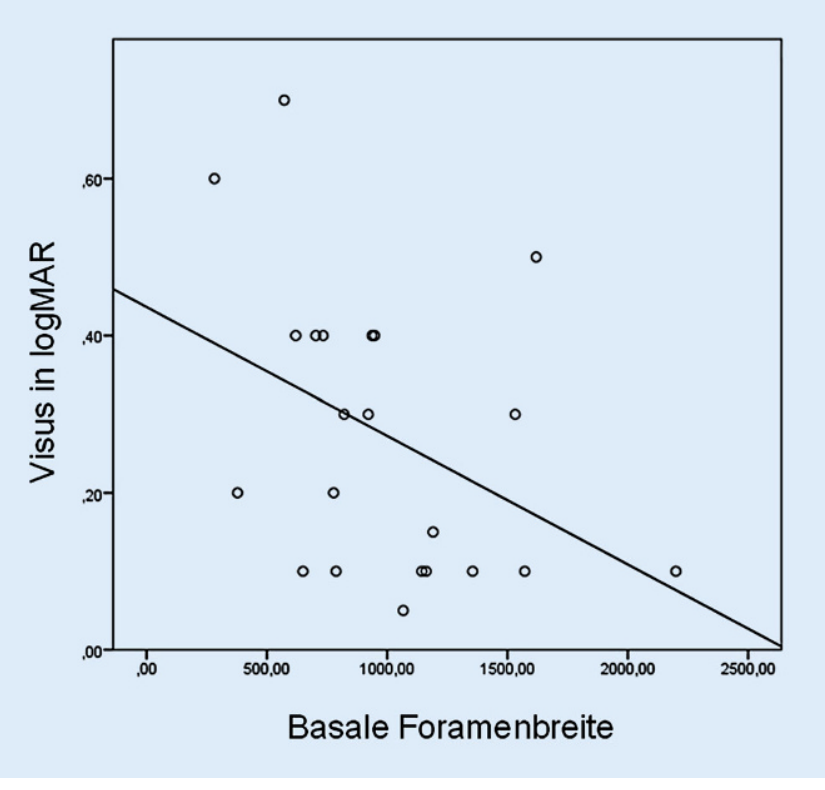

Abb. 4 ム Korrelation von intraoperativer basaler Foramenbreite nach ILMMembranpeeling und postoperativem Visusergebnis (mindestens 6 Monate postoperativ)

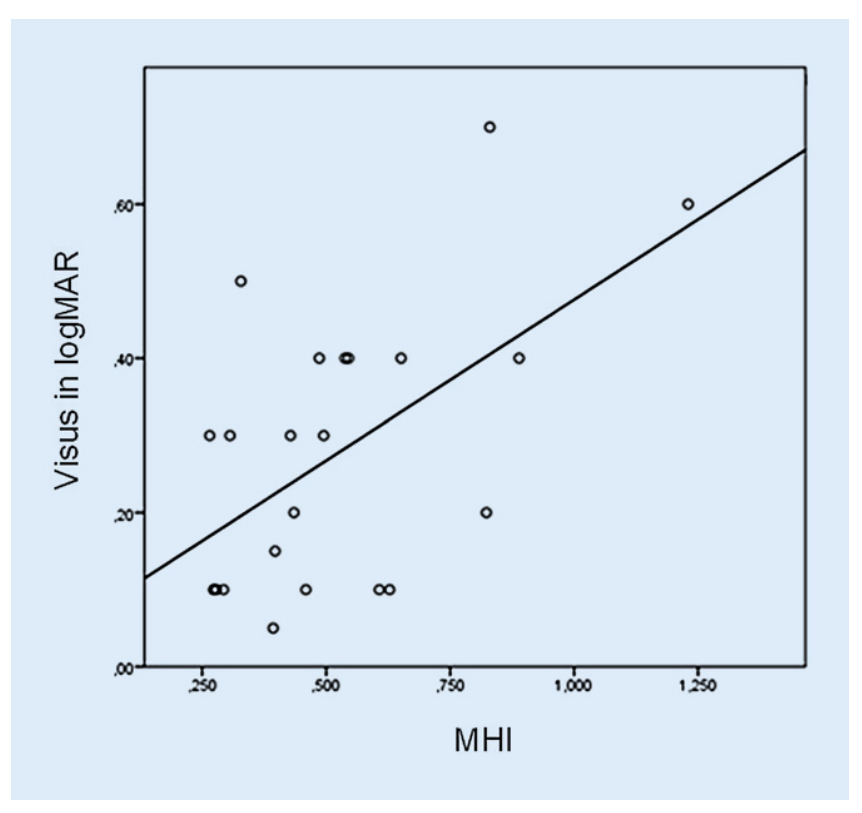

Abb. 5 A Korrelation von intraoperativem MHInach ILM-Membranpeeling und postoperativem Visusergebnis (mindestens 6 Monate postoperativ)
Unsere Kohorte zeigte eine gleichmäßige Größenverteilung des MF (klein, mittel, groß). Dennoch muss in Betracht gezogen werden, dass Patienten mit einem großen MF präoperativ einen geringeren Visus aufweisen als Patienten mit einem kleinen Makulaforamen. Das Visusverbesserungspotenzial ist bei Patienten mit großem MF demnach, statistisch gesehen, größer als bei Patienten mit kleinem Makulaforamen, wobei ein präoperativ großes Makulaforamen als negativer prognostischer Marker gilt.

Ein iOCT-integriertes Messinstrument zur Analyse der MF-Morphologie in Echtzeit stand dem Operateur nicht zur Verfügung.

Unsere Arbeit zeigt die Möglichkeit der Visualisierung von intraoperativen Veränderungen der Netzhautmorphologie mittels iOCT in der klinischen Anwendung. Eine intraoperative Anpassung der therapeutischen Strategie ist dadurch möglich geworden $[5,15,17,20]$.

\section{Fazit für die Praxis}

- Dank der IOCT wird die intraoperative Beobachtung der Netzhautmorphologie und deren Veränderung bei Patienten mit durchgreifendem Makulaforamen möglich.
- Während der Operation zeigt sich eine Entspannung der Netzhaut, welche sich in einer Breitenzunahme und einer Höhenabnahme des Makulaforamens darstellt.

- Eine mobile Netzhaut am Ende der Operation steht in Zusammenhang mit einem besseren postoperativen Visus.

\section{Korrespondenzadresse}

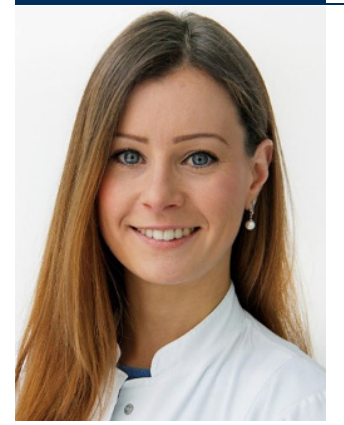

\section{Dr. med. Julia Sabina Friedrich}

Klinik und Poliklinik für Augenheilkunde,

Klinikum rechts der Isar, Technische Universität München

Ismaningerstr. 22, 81675 München,

Deutschland

Julia.Friedrich@mri.tum.de

Funding. Open Access funding enabled and organized by Projekt DEAL.

\section{Einhaltung ethischer Richtlinien}

Interessenkonflikt. J.S. Friedrich, N. Bleidißel, A. Nasseri, N. Feucht, J. Klaas, C.P. Lohmann und M. Maier geben an, dass kein Interessenkonflikt besteht.

Für diesen Beitrag wurden von den Autoren keine Studien an Menschen oder Tieren durchgeführt. Für die aufgeführten Studien gelten die jeweils dort angegebenen ethischen Richtlinien.

Open Access. Dieser Artikel wird unter der Creative Commons Namensnennung 4.0 International Lizenz veröffentlicht, welche die Nutzung, Vervielfältigung Bearbeitung, Verbreitung und Wiedergabe in jeglichem Medium und Format erlaubt, sofern Sie den/die ursprünglichen Autor(en) und die Quelle ordnungsgemäß nennen, einen Link zur Creative Commons Lizenz beifügen und angeben, ob Änderungen vorgenommen wurden.

Die in diesem Artikel enthaltenen Bilder und sonstiges Drittmaterial unterliegen ebenfalls der genannten Creative Commons Lizenz, sofern sich aus der Abbildungslegende nichts anderes ergibt. Sofern das betreffende Material nicht unter der genannten Creative Commons Lizenz steht und die betreffende Handlung nicht nach gesetzlichen Vorschriften erlaubt ist, ist für die oben aufgeführten Weiterverwendungen des Materials die Einwilligung des jeweiligen Rechteinhabers einzuholen.

Weitere Details zur Lizenz entnehmen Sie bitte der Lizenzinformation auf http://creativecommons.org/ licenses/by/4.0/deed.de.

\section{Literatur}

1. Bleidißel N, Friedrich J, Klaas J et al (2021) Inverted internal limiting membrane flap tech- 
nique in eyes with large idiopathic full-thickness macular hole: long-term functional and morphological outcomes. Graefes Arch Clin Exp Ophthalmol 259(7):1759-1771. https://doi.org/ 10.1007/s00417-021-05082-7

2. Carl Zeiss Meditec Technische Daten (2017) Available from: https://www.zeiss.de/ meditec/produkte/ophthalmologie/katarakt/ visualisierung/operationsmikroskope/opmilumera-700.html\#technische-daten. Zugegriffen: 13.11.2017

3. Dayani PN, Maldonado R, Farsiu S et al (2009) Intraoperative use of handheld spectral domain optical coherence tomography imaging in macular surgery. Retina 29:1457-1468

4. Duker JS, Kaiser PK, Binder S et al (2013) The international vitreomacular traction study group classification of vitreomacular adhesion, traction, and macular hole. Ophthalmology 120:2611-2619

5. Ehlers JP, Goshe J, Dupps WJ et al (2015) Determination of feasibility and utility of microscopeintegrated optical coherence tomography during ophthalmic surgery: the DISCOVER study RESCAN results. JAMA Ophthalmol 133:1124-1132

6. Ehlers JP, Kaiser PK, Srivastava SK (2014) Intraoperative optical coherence tomography using the RESCAN 700: preliminary results from the DISCOVER study. Br J Ophthalmol 98:1329-1332

7. Ehlers JP, Uchida A, Srivastava SK et al (2019) Predictive model for macular hole closure speed: insights from intraoperative optical coherence tomography. TransI Vis Sci Technol 8:18

8. Ehlers JP, Xu D, Kaiser PK et al (2014) Intrasurgical dynamics of macular hole surgery: an assessment of surgery-induced ultrastructural alterations with intraoperative optical coherence tomography. Retina 34:213-221

9. Friedrich J, Bleidißel N, Klaas J et al (2021) Large macular hole - always a poor prognosis? Ophthalmologe 118(3):257-263

10. Fujimoto J, Swanson E (2016) The development, commercialization, and impact of optical coherence tomography. Invest Ophthalmol Vis Sci 57(9):OCT1-OCT13

11. Gupta B, Laidlaw DA, Williamson TH et al (2009) Predicting visual success in macular hole surgery. Br JOphthalmol 93:1488-1491

12. Hattenbach LO, Framme C, Junker B et al (2016) Intraoperative real-time OCT in macular surgery. Ophthalmologe 113:656-662

13. Hocaoglu M, Muslubas IS, Ersoz MG et al (2018) First-operated and fellow eyes with bilateral idiopathic macular hole: comparison of anatomical and functional postoperative outcomes. Ophthalmic Surg Lasers Imaging Retina 49:571-578

14. Kusuhara S, Teraoka Escano MF, Fujii $S$ et al (2004) Prediction of postoperative visual outcome based on hole configuration by optical coherence tomography in eyes with idiopathic macular holes. Am J Ophthalmol 138:709-716

15. Maier M, Bohnacker S, Klein J et al (2019) Vitrectomy and IOCT-assisted inverted ILM flap technique in patients with full thickness macular holes. Ophthalmologe 116(7):617-624

16. Maier M, Schumann R, Friedrich J et al (2021) Biomarkers in full-thickness and lamellar defects of the macula. Ophthalmologe 118:321-336

17. Maier MM, Nasseri A, Framme C et al (2018) Intraoperative optical coherence tomography in vitreoretinal surgery: clinical experiences and future developments. Klin Monatsbl Augenheilkd 235:1028-1034
IOCT in clinical use. Correlation of intraoperative morphology and postoperative visual outcome in patients with full thickness macular hole

Background: Due to intraoperative optical coherence tomography (iOCT), observation of retinal morphological changes during surgery has become possible.

Objective: To analyze the intraoperative morphology of full thickness macular holes (FTMH) and the correlation with the postoperative function, a retrospective, observational clinical study was performed analyzing 32 eyes of patients treated at the hospital of the technical university of Munich.

Material and methods: Using IOCT in 32 eyes of 32 consecutive patients, the operative morphology was analyzed during surgery. These findings were then correlated with the postoperative visual outcome.

Results: After posterior vitreous detachment (PVD) the macular hole index (MHI) decreased by $-0.05(p=0.01)$ and the base diameter (BD) increased by $+99.4 \mu \mathrm{m}$ $(\mathrm{SD}=197.8 \mu \mathrm{m} ; p=0.04)$. Closure rate was $100 \%$ at the first visit after a mean time of 73 days and the postoperative best corrected visual acuity (BCVA) significantly improved $(p<0.05)$.

There were significant correlations between intraoperative morphology and postoperative results indicating a relation between low $\mathrm{MHI}$ and better postoperative $\mathrm{BCVA}(\mathrm{SCC}=0.50 ; p=0.02)$, large $\mathrm{BD}$ and better postoperative $\mathrm{BCVA}(\mathrm{SCC}=0.43$; $p=0.05)$ and large aperture after PVD and higher improvement of $\mathrm{BCVA}(\mathrm{SCC}=0.44$; $p=0.03$ ).

Conclusion: Flattening and broadening of the FTMH occurred as a result of reduction of vitreoretinal traction. The significant correlation between a large operative $B D$ and improved BCVA reveals the importance of intraoperative retinal relaxation.

\section{Keywords}

IOCT · Operative Imaging · Vitreoretinal Surgery · Macular Hole Surgery · SD-OCT

18. Meyer CH, Borny R, Horchi N (2017) Subretinal fluid application to close a refractory full thickness macular hole. Int J Retin Vitr 3:44

19. Rossi T, Gelso A, Costagliola C et al (2017) Macular hole closure patterns associated with different internal limiting membrane flap techniques. Graefes Arch Clin Exp Ophthalmol 255:1073-1078

20. Stanzel BV, Gagalick A, Brinkmann CK et al (2016) Intraoperative OCT in ophthalmic microsurgery. Ophthalmologe 113:435-442

21. Ullrich S, Haritoglou C, Gass Cet al (2002) Macular hole size as a prognostic factor in macular hole surgery. Br J Ophthalmol 86:390-393 